\title{
Intramural Hematoma of the Ascending Aorta
}

\author{
Noedir Antônio G. Stolf, Anderson Benício, Gustavo I. Judas, Roberto Rocha Correia Veiga Giraldez, Wilson \\ Mathias Júnior \\ Instituto do Coração do Hospital das Clínicas - FMUSP - São Paulo, SP, Brazil
}

We report the case of a 71-year-old male patient who was admitted to the emergency room with pallor of the skin and mucous membranes accompanied by systemic hypertension and chest pain. Diagnostic investigation did not show any alteration compatible with acute myocardial ischemia. Chest radiography showed a significant enlargement of the mediastinum. Echocardiogram revealed a $47-\mathrm{mm}$ ascending aorta at the pulmonary trunk. One day after the echocardiogram was performed the patient underwent a magnetic resonance imaging (MRI) that showed a 62-mm ascending aorta, with no evidence of flow within the false lumen or intimal flap, but with an intramural hematoma of the ascending aorta which extended from the aortic root to the proximal third of the aortic arch. Surgical correction was performed with replacement of the ascending aorta and part of the aortic arch (hemiarch), with aortic valve-sparing with commissural resuspension. The patient recovered uneventfully and was discharged in the ninth postoperative day. In this case report we emphasize the similarity between the clinical presentation of aortic intramural hematoma and that of aortic dissection, the importance of a correct diagnosis and the best treatment.

Intramural hematoma of the aorta $(\mathrm{IMH})$ is an acute aortic disease caused by a hemorrhage within the aortic wall, but, unlike classic acute dissection (AD), without an evident tear in the intima or flow in the false lumen ${ }^{1}$. Historically, its origin is attributed to the rupture of the vasa vasorum within the aortic wall ${ }^{1}$. However, some authors have used the term to define this condition as a possible complication of an intimal rupture (which cannot be visualized) due to a penetrating ulcer of the aortic wall².

These hematomas, like aortic dissections, involve the ascending aorta, aortic arch or both (type A), or the descending aorta (type B). Clinical presentations of patients with aortic dissection and its variant forms, among them the $\mathrm{IMH}$, are indistinguishable ${ }^{3}$.

Currently, IMHs account for at least $6 \%$ of all non-traumatic acute aortic syndromes and in some autopsy studies they account for $5 \%$ to $13 \%$ of the total number of these cases ${ }^{4}$.

The natural history of IMHs may involve progression to rupture and or dissection, or regression with resolution of the lesion. Many authors believe that IMH is a step in the progression to aortic dissection ${ }^{3,5}$.

Management of this condition varies according to its location; however there is no consensus as to early surgical indication for patients with type-A lesions in whom no complicating factors such as hemodynamic instability, pain, rupture, imminent rupture or signs of cardiac tamponade are found.

This article reports the case of a patient with a complicated intramural hematoma of the ascending aorta who underwent a successful surgical treatment.

\section{Case Report}

Seventy-one-year-old male patient admitted to the hospital with a history of severe chest pain radiating to the cervical region, with a sudden onset and stabbing character, accompanied by nausea and low-grade fever. He reported systemic hypertension inadequately controlled.

Chest radiography showed enlargement of the cardiac silhouette and mediastinum. No significant alterations were observed in the electrocardiogram. Transesophageal echocardiogram revealed aortic ectasia $(47 \mathrm{~mm})$ at the midascending aorta, mild aortic regurgitation with preserved ventricular function and moderate pericardial effusion. Magnetic resonance imaging (MRI) was performed one day after the echocardiogram, and showed a 62-mm intramural hematoma at the mid-ascending aorta (Fig. 1).

Cardiac catheterization evidenced a greater-than-90\% lesion in the anterior interventricular artery and a nonsignificant lesion in the first diagonal artery.

The patient then underwent conventional surgical treatment with extracorporeal circulation (ECC), and revascularization of the anterior interventricular branch, with a left internal thoracic artery graft and replacement of the ascending aorta by a 28$\mathrm{mm}$ Dacron tube were performed. Due to the extension of the hematoma into the aortic arch, the brachiocephalic trunk (BCT) was cannulated to perform the ECC. The patient was cooled down to $20^{\circ} \mathrm{C}$ and circulatory arrest with antegrade selective cerebral perfusion was started through the BCT. The aorta was opened, showing an intramural hematoma dissecting the media without intimal tear, extending from the sinuses of Valsalva up to the proximal third of the aortic arch.

\section{Key words}

Ascending aorta, hematoma.

Mailing Address: Anderson Benício •

Alameda Franca, 63/12 - 01422-000 - São Paulo, SP, Brazil

E-mail: benicio@cardiol.br 


\section{Case Report}

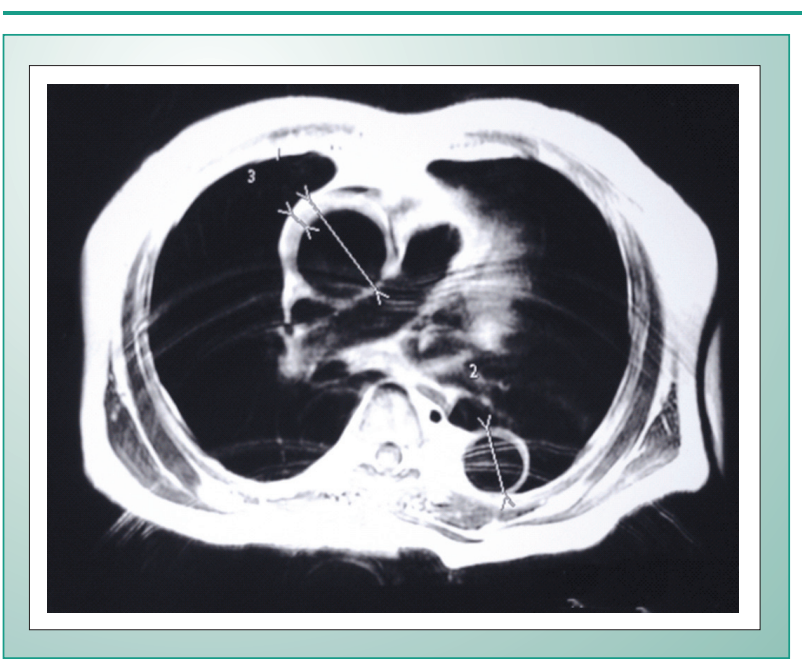

Fig. 1 - Magnetic resonance imaging. Hematoma of the ascending aorta showing the ascending aorta at the pulmonary trunk. 1- Aorta with a 62-mm diameter: 2 - descending thoracic aorta with a 35- $\mathrm{mm}$ diameter albeit still with a mild hematoma within its wall; 3 - ascending aorta with an 11- $\mathrm{mm}$ wall thickness characterizing the hematoma of the aortic wall.

The delamination in the proximal and distal stump was corrected with Dacron bands inside and outside the aorta, with further suturing of the tube (interposition of a $28-\mathrm{mm}$ Dacron graft).

The patient recovered uneventfully and was discharged in the ninth postoperative day.

\section{Discussion}

The first description of aortic intramural hematoma in the literature dates back to 1920, when a spontaneous intramural hematoma of the aortic wall without an intimal flap was demonstrated $^{4}$. Since then, approximately two hundred cases, most of which involving the descending aorta, were described in the literature, and most of the studies describing $\mathrm{IMH}$ have analyzed small case series ${ }^{4}$

There is a higher prevalence in males $(61 \%)$ with a mean age for both males and females of 63 and 68 years, respectively, and previous history of systemic hypertension in $53 \%$. The non-traumatic form is the most frequent, with a $94 \%$ incidence. Aortic regurgitation and pulse deficits are less frequent in IMH than in aortic dissection. Symptoms such as chest and/or back pain were present in more than $80 \%$ of the cases ${ }^{2}$. This symptom is also the most common finding in dissection cases, and the time for the onset of symptoms is similar for both conditions. Differences regarding clinical presentation refer to the time required for the diagnosis of the disease and a greater number of tests required in $\mathrm{IMH}$ in relation to the classic cases of acute dissection ${ }^{4}$.

The possibility of progression of type A IMH to dissection with cardiac tamponade or aortic rupture in some patients medically treated is well known. Studies have demonstrated that most often such progression occurs within a period of up to seven days from the onset of symptoms ${ }^{4,5}$.

As regards the diagnostic methods used, transesophageal echocardiogram represents a very fast and easy-to-perform diagnostic tool with a sensitivity, specificity and accuracy between $90 \%$ and $99 \%{ }^{4}$.

Computed tomography (CT) scan is a valuable diagnostic method that can evidence a weakness in the wall and differentiate it from intramural thrombosis. The advantage of the CT scan over the echocardiogram is a better visualization of vessels of the aortic arch and their relation with IMH or AD. However, the use of a contrast medium is a disadvantage ${ }^{4}$.

MRI is an additional imaging method that can be used in the diagnosis of $\mathrm{IMH}$. Subacute hematomas with formation of methemoglobin produce increased signal intensity in T1weighted images. However, it may be more difficult to detect acute cases of IMH which can mimic an atherosclerotic process in the vessel wall ${ }^{4}$.

In hematomas, aortography is not the most appropriate diagnostic method. The image is always negative because of the lack of communication between the false and the true lumen.

The high risk of a non-surgical management of type A hematoma is reflected in the $55 \%$ early mortality rate with pharmacological treatment (approximately 20\% progress to dissection). Considering global data on early mortality, the rate is of $12 \%$ for surgical treatment and $24 \%$ for pharmacological treatment. These data corroborate a tendency for a better prognosis with surgery ${ }^{6}$.

Recent Asian studies, however, have reported lower mortality rates - of approximately $7 \%$ - with pharmacological treatment ${ }^{6}$. One of the factors that could explain this difference is the early diagnosis with prompt pharmacological treatment. In addition, a close monitoring of these patients regarding progression to dissection and an occasional need for surgical intervention did not result in increased mortality ${ }^{7}$.

The discrepancy between these data reveals the importance of establishing predictive factors of the progression of IMHs. A proximal hematoma involving the ascending aorta is an important predictor for the early progression to dissection, and a greater-than-50mm diameter in computed tomography or a greater-than-12 mm aortic wall thickness within two weeks after the onset of symptoms result in positive and negative predictive values of $80 \%$ and $100 \%$, respectively, ${ }^{8,9}$. Late progression (more than thirty days) is associated with young age, Marfan syndrome and absence of treatment with beta-blockers ${ }^{6,8,9}$.

Considering an older population and a higher mortality and morbidity of surgery of the aorta, we believe that the medical treatment in patients with intramural hematoma of the ascending aorta is still feasible. However, the option of surgical treatment should be considered, since survival in this group is higher when compared with medical treatment, even though this management still lacks more support from investigations with more representative case series. In the present report, the patient had a persistent pain and pericardial effusion that supported the surgical indication. In the ascending aorta, the operation consists of the replacement of this vessel segment whereas in the descending exist the possibility of the endovascular procedure.

\section{Potential Conflict of Interest}

No potential conflict of interest relevant to this article was reported. 


\section{References}

1. Isselbacher EM. Intramural hematoma of the aorta: should we let down our guard? Am J Med. 2001; 13: 244-6.

2. Maraj R, Rerkpattanapipat, P. Jacobs LE, Makornwattana P. Kotler M N. Metaanalysis of 143 reported cases of aortic intramural hematoma. Am J Cardiol. 2000; 86: 664-8.

3. Saborio DV, Sadeghi A, Burack J, Lowery RC, Genovesi MH, Brevetti GR. Intramural hematoma of the ascending aorta and aorta arch: the risk of limited surgery. Tex Heart Inst J. 2003; 30: 325-7.

4. Vaccari G, Caciolli S, Calamai G, Acquafresca M, Montesi G, Braconi L, et al. Intramural hematoma of the aorta: diagnosis and treatment. Eur J Cardiothorac Surg. 2001; 19: 170-3.

5. Kaji S, Akasaka T, Horibata Y, Nishigami K, Shono H, Katayama M, et al. Long-term prognosis of patients with type A aortic intramural hematoma. Circulation. 2002; 106 (12 Suppl I): I248-I252.
6. von Kodolitsch YV, Csösz SK, Koschyk DH, Schalwat I, Loose R, Karck M, et al. Intramural hematoma of the aorta. predictors of progression to dissection and rupture. Circulation. 2003; 107: 1158-63.

7. Song JK, Kim HS, Kang DH, Lim TH, Song MG, Park SW, et al. Different clinical features of aortic intramural hematoma versus dissection involving the ascending aorta. J Am Coll Cardiol. 2001; 37: 1604-10.

8. Moizumi Y, Komatsu T, Motoyoshi N, Tabayashi K. Clinical features and long-term outcome of type A and type B intramural hematoma of the aorta. J Thorac Cardiovasc Surg. 2004; 127: 421-7.

9. Evangelista A, Mukherjee D, Metha RH, O' Gara PT, Fattori R, Cooper JV, et al for the International Registry of Aortic Dissection (IRAD) Investigators. Acute intramural hematoma of the aorta. A mystery in evolution. Circulation. 2005; 111: 1063-70. 